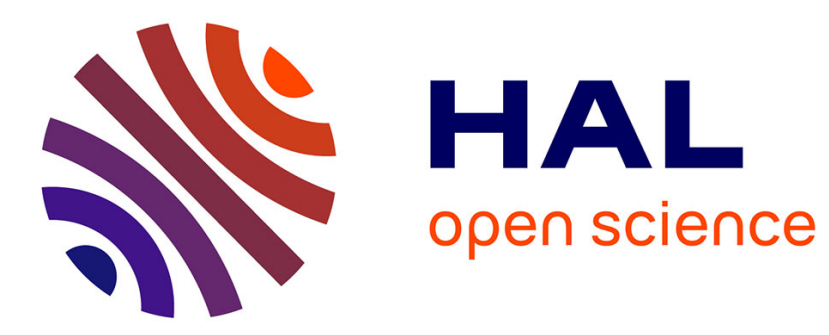

\title{
Measuring low amplitude periodical phase shift without set-up stabilization: application to Pockels effect characterization
}

Philippe Delaye, Gérald Roosen

\section{- To cite this version:}

Philippe Delaye, Gérald Roosen. Measuring low amplitude periodical phase shift without set-up stabilization: application to Pockels effect characterization. Optics Communications, 2002, 214, pp.199206. 10.1016/S0030-4018(02)02134-X . hal-00673942v2

\section{HAL Id: hal-00673942 \\ https://hal-iogs.archives-ouvertes.fr/hal-00673942v2}

Submitted on 30 Mar 2012

HAL is a multi-disciplinary open access archive for the deposit and dissemination of scientific research documents, whether they are published or not. The documents may come from teaching and research institutions in France or abroad, or from public or private research centers.
L'archive ouverte pluridisciplinaire HAL, est destinée au dépôt et à la diffusion de documents scientifiques de niveau recherche, publiés ou non, émanant des établissements d'enseignement et de recherche français ou étrangers, des laboratoires publics ou privés. 


\title{
Measuring low amplitude periodical phase shift without set-up stabilization: application to Pockels effect characterization
}

\author{
Philippe Delaye, Gérald Roosen
}

\begin{abstract}
Laboratoire Charles Fabry de l'Institut d'Optique, du Centre National de la Recherche Scientifique et de l'Université Paris Sud, Bat. 503, Centre Scientifique d'Orsay, 91403 Orsay Cedex, France.
\end{abstract}
Corresponding author: $\quad$ Philippe Delaye, Tel : 33-1-69-35-87-50, Fax : 33-1-69-35-87-00, Email : Philippe.delaye@iota.u-psud.fr

\begin{abstract}
:
We present an interferometric set-up for the measurement of low amplitude sinusoidal phase shifts that releases most of the constraints of usual architectures. It allows measurement without any stabilization of the set-up and can tolerate low quality fringe pattern. Due to its ease of use and its good sensitivity, this set-up can be used to measure low amplitude electro-optic coefficients with low applied voltage (less than $1 \mathrm{~V}$ ). Test measurements on $\mathrm{LiNbO}_{3}$ and $\mathrm{LiTaO}_{3}$ samples show good accordance between measured and theoretical Pockels phase shifts.
\end{abstract}

PACS : 42.65.Hz, 42.62.Eh, 77.84.Dy, 78.20.Jq

The measurement of the electro-optic coefficients of materials is an important problem of optoelectronic, especially when low Pockels coefficients or low thickness samples are measured. Several techniques are used, they are presented and discussed in Ref. [1-11]. Among them, interferometric techniques are very powerful as they can reach independently several coefficients in usual crystals. One problem with these techniques is that they require to put the interferometer to a peculiar fringe position (usually at the maximal slope of the fringes), and to stabilize it at this 
position. The experiment will thus be particularly sensitive to vibrations of the set-up and to thermal fluctuations of the studied crystal. In this letter we propose a new solution to this problem that works without the need of a stabilized interferometer and that even takes benefit of its drift and fluctuations for the measurement. This set-up thus enables to measure the very small phase shifts induced in Pockels crystals (such as $\mathrm{LiNbO}_{3}$ or $\mathrm{LiTaO}_{3}$ ) by AC voltage as low as some tens of $\mathrm{mV}$.

In the interferometric set-up, a laser beam is separated in two arms by a beam splitter and recombined on a detector (using the same or another beam splitter). In one of the arms (the signal beam), we place the sample to be measured, whereas in the second arm a piezo-mirror allows to slowly vary the phase shift between the beams of the interferometer. When the interferometer is aligned (i.e. fringes are observed), the intensity received by the detector equals:

$$
\mathrm{I}=\mathrm{I}_{\mathrm{S}}+\mathrm{I}_{\mathrm{R}}+2 \mathrm{r} \sqrt{\mathrm{I}_{\mathrm{S}} \mathrm{I}_{\mathrm{R}}} \cos \psi(\mathrm{t})
$$

where $I_{S}$ is the intensity of the signal beam, in which is placed the Pockels crystal to be characterized and $I_{R}$ is the intensity of the reference beam. In this expression, $r$ is a coefficient that renders the quality of the fringes observed on the detector. For perfectly aligned plane waves, we have $r=1$ meaning that the phase shift between the beams does not vary on the surface of the detector. In the worst case of a large number of fringes on the surface of the detectors, meaning that the wavefronts are distorted or misaligned, we have $r \approx 0$, and no modulation signal is delivered by the detector. In the general case, we have a value of $r$ that varies between these two extrema, with a value of $r$ that will have to be the closest as possible to 1 as the detected modulated signal is proportional to this value. Nevertheless, as we will see in the following, the technique we developed works whatever the quality of the fringes, and thus whatever the value of $r$ (remembering that the measurement will be all the more easy and accurate than $r$ will be close to 1 , as the measured signal will be high). Finally, $\psi(\mathrm{t})$ is the phase shift between the two interfering beams. It can be separated in two terms $\psi(\mathrm{t})=\varphi(\mathrm{t})+\Delta \varphi(\mathrm{t})$, with $\Delta \varphi(\mathrm{t})=\sqrt{2} \Delta \varphi_{\mathrm{RMS}} \sin (2 \pi \mathrm{ft})$ the sinusoidal Pockels phase shift that we want to measure and $\varphi(t)$ the mean phase shift between the beams that varies much slowly than the Pockels phase shift. The intensity measured by the detector can then be rewritten as (taking into account that for the measured phase shift we have $\Delta \varphi(\mathrm{t})<<\pi / 2)$ :

$$
I=\underbrace{I_{S}+I_{R}+2 r \sqrt{I_{S} I_{R}} \cos \varphi(t)}_{I_{\text {mean }}} \underbrace{-2 r \sqrt{I_{S} I_{R}} \Delta \varphi(t) \sin \varphi(t)}_{\Delta I(t)}
$$


In an usual interferometric set-up (homodyne detection) [1], the mean phase shift $\varphi(t)$ is stabilized to a constant value equal to $\pm \pi / 2$ (half fringe level), where the detected signal $\Delta \mathrm{I}(\mathrm{t})$ is maximal. This requires the knowledge of the fringes contrast (to determine the half level). This value is obtained through a measurement of the maximum of the mean intensity $\mathrm{I}_{\text {Mean }}$ reaching the detector (corresponding to $\varphi(t)=0$ ), and the minimum mean intensity (corresponding to $\varphi(t)=\pi$ ). This stabilization at half fringe level is the main problem of usual homodyne detection schemes. In fact, the problem is not necessarily the stabilization by itself, but to find the right level of stabilization that will vary slowly if $I_{R}, I_{S}$ or above all $r$ vary. This is generally obtained by a stabilization (against thermal change, vibrations, air fluctuations, ...) of the whole set-up during the measurement.

In our case, we use the same optical set-up and the same signal but we change the data processing. Our method does not require the stabilization of the interferometer to a given level, it will even use the naturally or artificially produced fluctuations of $\varphi(t)$, without the necessity to know the exact value of this phase shift. This method can be seen as a low frequency analogous to the heterodyne detection where the ratio of height of the sideband to the carrier frequency amplitude give directly the phase modulation amplitude.

Considering the intensity received by the detector given by equation (2), it can be split into two terms that evolve on very different time scales : a signal $\Delta \mathrm{I}(\mathrm{t})$ modulated at the excitation frequency of the Pockels material and a mean intensity $I_{\text {Mean }}$ that varies slowly in time but is constant at the evolution time scale of $\Delta \mathrm{I}(\mathrm{t})$. The detector sees a quasi-uniform tint of an interference pattern, and the mean intensity $\mathrm{I}_{\text {Mean }}$ is directly linked to the interference state of the fringe seen by the detector. The phase shift due to the drift of the set-up induces a displacement of the fringe pattern in front of the detector that corresponds to a change of the mean intensity $\mathrm{I}_{\text {Mean }}$ and a slow variation of the amplitude of the modulated signal $\Delta \mathrm{I}(\mathrm{t})$. The important point is here that the temporal variations of the mean intensity $\mathrm{I}_{\text {Mean }}$ and of the amplitude of the modulated signal $\Delta \mathrm{I}(\mathrm{t})$ are not independent and are correlated.

We will now show that if we measure simultaneously the RMS (Root Mean Square) value of the modulated signal $\Delta \mathrm{I}_{\mathrm{RMS}}$ (supposed in all this study to be sinusoidal) and the mean value of the intensity, we can deduce the RMS value of the Pockels phase shift we want to determine. From equation (2), we deduce:

$$
\Delta \mathrm{I}_{\mathrm{RMS}}=-2 \mathrm{r} \sqrt{\mathrm{I}_{\mathrm{S}} \mathrm{I}_{\mathrm{R}}} \sin \varphi(\mathrm{t}) \Delta \varphi_{\mathrm{RMS}}
$$

and 


$$
I_{\text {Mean }}=I_{S}+I_{R}+2 r \sqrt{I_{S} I_{R}} \cos \varphi(t)
$$

In these relation, we immediately recognize the parametric equation of an ellipse $(\varphi(t)$ being the parameter), centered in the point $\left(0, I_{S}+I_{R}\right)$ with a small axis of length $4 r \sqrt{I_{S} I_{R}} \Delta \varphi_{R M S}$ and a great axis of length $4 r \sqrt{I_{S} I_{R}}$. The ratio of the length of the two axes of this ellipse gives the information we want, i.e. $\Delta \varphi_{\text {RMS }}$. Nevertheless, the determination of the phase shift $\Delta \varphi_{\text {RMS }}$ through the ratio of the axes of the ellipse, only use a small part of the measured data (points around the extrema of the ellipse). The same parameter should be obtained with a better accuracy using an adjustment of the whole ellipse. As the adjustment with the ellipse equation in not really easy and common, we have choosen another treatment, that will give more easily the same result, i.e. the determination of $\Delta \varphi_{\mathrm{RMS}}$ using all the measured data.

Eliminating the slow phase shift $\varphi(t)$ from equations (3) and (4) (calculating $\cos ^{2} \varphi(t)$ and $\left.\sin ^{2} \varphi(t)\right)$, we arrive to the relation:

$$
\Delta \mathrm{I}_{\mathrm{RMS}}^{2}=\underbrace{-\Delta \varphi_{\mathrm{RMS}}^{2}}_{\mathrm{a} 1} \mathrm{I}_{\text {Mean }}^{2}+\underbrace{2 \Delta \varphi_{\mathrm{RMS}}^{2}\left(\mathrm{I}_{\mathrm{S}}+\mathrm{I}_{\mathrm{R}}\right)}_{\mathrm{a} 2} \mathrm{I}_{\text {Mean }}+\underbrace{\Delta \varphi_{\mathrm{RMS}}^{2}\left(4 \mathrm{r}^{2} \mathrm{I}_{\mathrm{S}} \mathrm{I}_{\mathrm{R}}-\left(\mathrm{I}_{\mathrm{S}}+\mathrm{I}_{\mathrm{R}}\right)^{2}\right)}_{\mathrm{a} 3}
$$

We easily see that the graphical representation of this expression $\Delta \mathrm{I}_{\mathrm{RMS}}^{2}=\mathrm{f}\left(\mathrm{I}_{\text {Mean }}\right)$ is a parabola, which a1 parameter directly gives the RMS value $\Delta \varphi_{\mathrm{RMS}}$ of the phase shift $\Delta \varphi(\mathrm{t})$ due to the voltage applied to the Pockels crystal. This measure is completely independent of the intensity of the different beams $\left(I_{S}\right.$ and $I_{R}$ ), but also of the quality of the fringes (characterized by parameter $\mathrm{r})$. Moreover the exact nature of the slow phase shift $\varphi(t)$ is not important. It does not need to vary according to a peculiar law (it can even be due to the natural drift of the interferometer) as soon as at least one fringe is scrolled $(\varphi(t)>\pi)$, and the whole parabola is described by the signal with typically some cycles (two or three are usually sufficient).

Experimentally, the interferometer is of the Mach-Zehnder type, using a collimated and vertically polarized He-Ne laser emitting at $633 \mathrm{~nm}$ (Figure 1). The Pockels crystal is placed in the signal arm. A half wave plate allows to choose the desired eigen-polarization of the crystal, a second half wave plate gives back a vertical polarization, selected by a second polarizer. At the output of the interferometer a lens makes the image of the crystal on the detector plane. The slow phase shift is created by a piezo-mirror inserted in the reference beam. The typical modulation frequency of the piezo-mirror is $20 \mathrm{mHz}$ with a displacement amplitude around $1 \mu \mathrm{m}$. The detected signal is sent simultaneously on one input of an oscilloscope (with an internal low pass filter) to measure the 
mean intensity and on the input of a lock-in amplifier to measure the RMS value of the detected signal at the excitation frequency (the results of the measurement being sent on the other input of the oscilloscope). The Lock-In amplifier is also used to deliver the voltage applied to the Pockels crystal. Its RMS amplitude varies typically between $10 \mathrm{mV}$ and $2 \mathrm{~V}$, with a frequency varying between $1 \mathrm{kHz}$ and $100 \mathrm{kHz}$ (high frequency limitation of the Lock-In amplifier).

Typical signals are shown in Figure 2a. We see the mean signal that varies as the fringe is scrolled in front of the detector. In the same time the RMS value of the signal varies (visually in quadrature) with a maximum value at half fringe height and zero signal for absolute maximum and minimum of the mean intensity as expected. We see that tracing $\Delta I_{R M S}=f\left(I_{\text {Mean }}\right)$, we obtain an ellipse as expected (Fig. 2b). After the treatment (tracing $\Delta \mathrm{I}_{\mathrm{RMS}}^{2}=\mathrm{f}\left(\mathrm{I}_{\text {Mean }}\right)$ ) we obtain the expected parabola (Fig. 3), which fit allows to measure a Pockels phase shift of $\Delta \varphi_{\text {RMS }}=13.047 \mathrm{mrad}$, with an accuracy given by a standard deviation of $3 \mu \mathrm{rad}$ (in the following the standard deviation will be used as a measure of the accuracy).

We used this set-up to characterize two reference Pockels crystals of $\mathrm{LiNbO}_{3}$ and $\mathrm{LiTaO}_{3}$. These crystals are of the same class of symmetry (the $3 \mathrm{~m}$ class). All their coefficients (electro-optic, elasto-optic, piezo-electric) are perfectly known and will be used to calculate the expected phase shift that will be compared to the experimental data given by the set-up. The crystals are cut along the crystallographic axes and are used in a transverse configuration with an electric field applied along the c-axis. The propagation is along the a-axis and the beam is either ordinary (i.e. vertically polarized along the b-axis) or extraordinary polarized (i.e. along the c-axis). The phase shift ( $\Delta \phi_{\mathrm{o}}$ and $\Delta \phi_{\mathrm{e}}$ for ordinary and extraordinary polarization respectively) acquired by the beam is then [12, 13]:

$$
\begin{aligned}
& \Delta \phi_{\mathrm{o}}=\frac{\pi}{\lambda} \mathrm{V}\left(\frac{\mathrm{L}}{\mathrm{d}}\right)\left[2\left(\mathrm{n}_{\mathrm{o}}-1\right) \mathrm{d}_{31}+\mathrm{n}_{\mathrm{o}}^{3} \mathrm{r}_{13}^{\mathrm{T}}\right] \\
& \Delta \phi_{\mathrm{e}}=\frac{\pi}{\lambda} \mathrm{V}\left(\frac{\mathrm{L}}{\mathrm{d}}\right)\left[2\left(\mathrm{n}_{\mathrm{e}}-1\right) \mathrm{d}_{31}+\mathrm{n}_{\mathrm{e}}^{3} \mathrm{r}_{33}^{\mathrm{T}}\right]
\end{aligned}
$$

where $\mathrm{V}$ is the applied voltage, $\mathrm{L}$ the thickness of the crystal along the beam propagation direction, $\mathrm{d}$ is the inter-electrode distance, and $\lambda$ the used wavelength in vacuum. In these expressions the first term is due to the change of the dimension of the crystal because of the piezo-electric effect (with the piezo-electric coefficient $\mathrm{d}_{31}$ ). The second term is due to the Pockels effect (with the unclamped electro-optic coefficient $r_{i j}^{T}$ ). In both cases, $n_{o}$ is the ordinary refractive index and $n_{e}$ is the 
extraordinary refractive index. These expressions suppose that the crystal is free, i.e. that the frequency used is low compared to the piezo-electric resonance. The values of these parameters in the crystals we used, are summarized in Table 1.

The first measurement is performed on a $\mathrm{LiNbO}_{3}$ crystal at a fixed frequency as a function of the applied electric field, for both polarizations (Figure 4). We observed a linear dependence of the measured phase shift with a slope equal to $\Delta \phi_{\mathrm{o}} / \mathrm{V}=(2.186 \pm 0.003) \mathrm{mrad} \cdot \mathrm{V}^{-1}$ and $\Delta \phi_{\mathrm{e}} / \mathrm{V}=(6.305 \pm 0.003) \operatorname{mrad} . \mathrm{V}^{-1}$. These experimental values are in good accordance with the theoretical ones given in Table 1. In the insert of Figure 4, the variation of the phase shift with the applied voltage is showed in a log-log scale. This presentation shows that the linearity of the variation extends on the whole measurement range (more than two decades), until applied voltage as low as $10 \mathrm{mV}$, corresponding to measured phase shifts as low as some tens of $\mu \mathrm{rad}$. We also measure the response as a function of the frequency of the applied sinusoidal voltage (Figure 5). The sensitivity (i.e. the measured RMS phase shift normalized to the RMS applied voltage) is constant on the whole frequency range with a small increase that appears above $30 \mathrm{kHz}$. In this range (i.e. below $30 \mathrm{kHz}$ ), the mean value of the sensitivity is $6.31 \pm 0.06 \mathrm{mrad} . \mathrm{V}^{-1}$, corresponding to a measurement accuracy of $1 \%$. The increase corresponds to the beginning of a piezoelectric resonance situated at higher frequency (theoretically around $600 \mathrm{kHz}$ according to the dimensions and the orientation of the crystal). This behaviour confirms that the crystal is free in the condition of the measurement what justifies the expressions (equations (6) and (7)) used for the phase shift.

Similar measurements were performed in three $\mathrm{LiTaO}_{3}$ crystals, with identical cut and dimensions. The obtained curves were similar to the one observed in $\mathrm{LiNbO}_{3}$, with nevertheless a slight dispersion of the sensitivity of the different crystals (Table 2) for the measurement at 25.7 $\mathrm{kHz}$. Despite this dispersion the obtained values are in accordance with the theoretical value (Table 1). This dispersion was attributed to some problem in the application of the electric field, but considered independent of the measurement set-up. Indeed, a similar dispersion exists for both extraordinary and ordinary polarization. In all the crystals the ratio of the extraordinary phase shift to the ordinary one, is the same at a value of $3.66 \pm 0.05$ that corresponds to the theoretical ratio. This point is confirmed by the fact that a measurement at lower frequency $(183 \mathrm{~Hz})$ shows a lower dispersion in the measured values with ordinary polarization (Table 2). For some samples and polarisations, measurement had been performed several times on different days, each time putting on and removing the sample from the set-up, with a new alignment and optimization for each measurement. For example for the sample LTO1 with ordinary polarization at the frequency of 
$51.2 \mathrm{kHz}, 5$ independent measurements were made giving sensitivities of $1.003,0.998,1.008,1.010$ and $1.013 \mathrm{mrad} . \mathrm{V}^{-1}$, what corresponds to a mean value of $1.006 \pm 0.006 \mathrm{mrad} . \mathrm{V}^{-1}$, i.e. a relative accuracy of $0.6 \%$ typical of the measurement performed.

The presented Pockels material characterization set-up has several advantages compared to conventional set-ups. It is simple to use and does not require any stabilisation of the set-up. It is not sensitive to external perturbations such as vibrations or thermal effects in the measured sample. It is an interferometric geometry, so contrarily to the other simple and stabilisation independent set-up, the Sénarmont set-up, it can by proper cut of the crystal allow the measurement of diverse electrooptic coefficients independently. The sensitivity of the measurement (some tens of $\mu$ rad without any peculiar precautions) allows to measure low amplitude electro-optic coefficients (such as $r_{13}^{T}$ in $\mathrm{LiNbO}_{3}$ or $\mathrm{r}_{41}^{\mathrm{T}}$ in InP) without the need to apply high voltage (some volts are sufficient). The measurement does not require very high fringe quality, even if a higher contrast will lead to higher signal and easier and more accurate measurement (see Appendix). The ultimate performances of the presented set-up should be the same than the one obtained with a classical stabilized interferometric set-up perfectly optimized and aligned. Nevertheless these performances are accessible more easily with the proposed set-up, without the need of a servo-controlled stabilization, a vibration isolated breadboard, an air flow protection or a thermal control. It can be a rather cheap and good solution for people having occasionnal needs for moderately accurate phase modulation measurements (caracterization of a phase modulator, determination of electro-optic or piezo-electric coefficients of a crystal, ... ), just using classical optical elements that are usually found in an optic laboratory.

\section{Appendix : Accuracy of the measurement technique}

The accuracy of the measurement technique can be theoretically estimated, using the fact that the phase shift is given by the ratio of the length of the axes to the ellipse when we trace $\Delta \mathrm{I}_{\mathrm{RMS}}=\mathrm{f}\left(\mathrm{I}_{\text {Mean }}\right)$. The axes are given by $\mathrm{a}=4 \mathrm{r} \sqrt{\mathrm{I}_{\mathrm{S}} \mathrm{I}_{\mathrm{R}}} \Delta \varphi_{\mathrm{RMS}}$ and $\mathrm{b}=4 \mathrm{r} \sqrt{\mathrm{I}_{\mathrm{S}} \mathrm{I}_{\mathrm{R}}}$. These parameters can be rewritten as a function of some other practical parameters of the fringe pattern, i.e. the maximum $\mathrm{I}_{\text {Mean }}^{\mathrm{Max}}$ or the minimum $\mathrm{I}_{\text {Mean }}^{\mathrm{Min}}$ intensity value, or the contrast $\mathrm{C}$ of the fringe pattern. For example, we have $\mathrm{b}=\mathrm{I}_{\text {Mean }}^{\text {Max }}-\mathrm{I}_{\text {Mean }}^{\text {Min }}=2 \mathrm{CI}_{\text {Mean }}^{\text {Max }} /(1+\mathrm{C})$.

The relative accuracy on the measured phase shift is given by 


$$
\frac{\Delta\left(\Delta \varphi_{\mathrm{RMS}}\right)}{\Delta \varphi_{\mathrm{RMS}}}=\frac{\Delta \mathrm{a}}{\mathrm{a}}+\frac{\Delta \mathrm{b}}{\mathrm{b}}
$$

We will now estimate these different terms. We have first :

$$
\frac{\Delta \mathrm{b}}{\mathrm{b}}=\frac{\Delta \mathrm{I}_{\text {Mean }}^{\mathrm{Max}}+\Delta \mathrm{I}_{\text {Mean }}^{\text {Min }}}{\mathrm{I}_{\text {Mean }}^{\text {Max }}-\mathrm{I}_{\text {Mean }}^{\text {Min }}}=\frac{\Delta \mathrm{I}_{\text {Mean }}(1+\mathrm{C})}{\mathrm{I}_{\text {Mean }}^{\text {Max }} \mathrm{C}}
$$

where we have considered that $\Delta \mathrm{I}_{\text {Mean }}^{\text {Min }} \approx \Delta \mathrm{I}_{\text {Mean }}^{\text {Max }}=\Delta \mathrm{I}_{\text {Mean }}$, is the standard deviation of the noise on the mean intensity. The relative error on $b$ is independent of the measured phase shift, as $b$ does not depend on it, but depends on the intensity and on the contrast of the fringes. The lower the intensity $\mathrm{I}_{\text {Mean }}^{\mathrm{Max}}$, the lower the signal and the worse the relative accuracy, where as for a low contrast the fringes disappear, the mean intensity variation becomes smaller and is determined with a less good accuracy.

Then, we have $\mathrm{a}=2 \Delta \mathrm{I}_{\mathrm{RMS}}^{\mathrm{Max}}$, with $\Delta \mathrm{I}_{\mathrm{RMS}}^{\mathrm{Max}}=2\left(\mathrm{I}_{\text {Mean }}^{\mathrm{Max}}-\mathrm{I}_{\text {Mean }}^{\mathrm{Min}}\right) \Delta \varphi_{\mathrm{RMS}}=4 \mathrm{CI}_{\text {Mean }}^{\mathrm{Max}} \Delta \varphi_{\mathrm{RMS}} /(1+\mathrm{C})$, from which we deduce :

$$
\frac{\Delta \mathrm{a}}{\mathrm{a}}=\frac{\Delta\left(\Delta \mathrm{I}_{\mathrm{RMS}}^{\mathrm{Max}}\right)}{\Delta \mathrm{I}_{\mathrm{RMS}}^{\mathrm{Max}}}=\frac{(1+\mathrm{C}) \Delta\left(\Delta \mathrm{I}_{\mathrm{RMS}}^{\mathrm{Max}}\right)}{4 \mathrm{CI}_{\text {Mean }}^{\mathrm{Max}} \Delta \varphi_{\mathrm{RMS}}}
$$

where $\Delta\left(\Delta \mathrm{I}_{\mathrm{RMS}}^{\mathrm{Max}}\right)$ is the noise on the output of the Lock-In amplifier giving the RMS value of the modulated signal. The relative error on "a" depends on the contrast of the fringes as a lower contrast means a smaller signal. It is the same for the maximum intensity of the fringe pattern that governs the measured modulated signal. The relative error also depends on the phase modulation amplitude as smaller phase modulation means smaller signal.

At small value of the measured phase modulation, the preponderant term for the relative accuracy is $\Delta \mathrm{a} / \mathrm{a}$, that decreases when phase modulation increases until saturation towards a value governed by the term $\Delta \mathrm{b} / \mathrm{b}$ at high values of the phase modulation.

In our experiment, typical values we measure, are $I_{\text {Mean }}^{\text {Max }}=100 \mathrm{mV}$ and a contrast of the fringes easily better than 0.5 . The performances of the detection is mainly governed by the electronic noise with typical values of $\Delta \mathrm{I}_{\text {Mean }} \approx 10 \mu \mathrm{V}$ and $\Delta\left(\Delta \mathrm{I}_{\mathrm{RMS}}^{\mathrm{Max}}\right) \approx 1 \mu \mathrm{V}$. The accuracy at large value of the phase modulation would reach $\Delta\left(\Delta \varphi_{\mathrm{RMS}}\right) / \Delta \varphi_{\mathrm{RMS}}=\Delta \mathrm{b} / \mathrm{b}=0.03 \%$, what correspond to the order of magnitude experimentally measured in our set-up (Fig. 3). 
At low amplitude of the phase modulation we can determine the smallest measurable phase shift with our set-up, i.e. the phase modulation RMS value that would lead to a relative accuracy $\Delta\left(\Delta \varphi_{\mathrm{RMS}}\right) / \Delta \varphi_{\mathrm{RMS}}=\Delta \mathrm{a} / \mathrm{a}=1$, equal to :

$$
\Delta \varphi_{\mathrm{RMS}}=\frac{(1+\mathrm{C}) \Delta\left(\Delta \mathrm{I}_{\mathrm{RMS}}^{\mathrm{Max}}\right)}{4 \mathrm{CI}_{\mathrm{Mean}}^{\mathrm{Max}}}
$$

giving a numerical value of $7.5 \mu \mathrm{rad}$, for our set-up. The order of magnitude of this value corresponds to the smallest value we were able to measure experimentally (i.e. $22 \mu \mathrm{rad}$ in Fig. 4).

\section{References}

[1] M.Aillerie, N. Théoufanous, M.D. Fontana. "Measurement of the electro-optic coefficients: description and comparison of the experimental techniques". Appl. Phys. B70, 317 (2000). [2] K.Onuki, N. Uchida, T.Saku. "Interferometric method for measuring electro-optic coefficcients in crystals". J. Opt. Soc. Am. 62, 1030 (1972).

[3] J.A. de Toro, M.D. Serrano, A. Garcia Cabanes, J.M. Cabrera. "Accurate interferometric measurement of electro-optic coefficicents: application to quasi-stoichiometric $\mathrm{LiNbO}_{3}$ ". Opt. Commun. 17, 332 (1976).

[4] P.Ney, A. Maillard, M.D. Fontana. "Accurate interferometric method for the measurement of electro-optic coefficients: application to a single B-barium borate crystal". J. Opt. Soc. Am. B17, 1158 (2000).

[5] Y. Fuji, T. Sakudo. "Interferometric determination of the quadratic electro-optic coefficient in $\mathrm{SrTiO}_{3}$ crystal". J. Appl. Phys. 41, 4118 (1970).

[6] K. Buse, F. Havemeyer, L. Glabasnia, K. Schlomp, E. Kratzig. "Quadratic polarization-optic coefficient of cubic $\mathrm{KTa}_{1-\mathrm{x}} \mathrm{Nb}_{\mathrm{x}} \mathrm{O}_{3}$ crystals". Opt. Commun. 131, 339 (1996).

[7] K. Clays, J.S. Schildkraut. "Dispersion of the complex electro-optic coefficient and electrochromic effect in poled polymer films". J. Opt. Soc. Am. B9, 2274 (1992).

[8] M.J. Shin, H.R. Sho, S.H. Han, J.W. Wu. "Analysis of the Mach-Zehnder interferometry measurement of the Pockels coefficients in a poled polymer film with a reflection configuration". J. Appl. Phys. 83, 1848 (1998). 
[9] Y. Lu, Z.Y. Cheng, S.E. Park, S.F. Liu, Q. Zhang. "Linear electro-optic effect of $0.88 \mathrm{~Pb}\left(\mathrm{Zn}_{1 / 3} \mathrm{~Pb}_{2 / 3}\right) \mathrm{O}_{3}-0.12 \mathrm{PbTiO}_{3}$ single crystal". Jpn. J. Appl. Phys. 39, 141 (2000).

[10] V.V. Spirin, C. Lee, K. No. "Measurement of the Pockels coefficient of lead zirconate titanate thin films by a two beam polarization interferometer with a reflection configuration". J. Opt. Soc. Am. B15, 1940 (1998).

[11] J.R. Fernandes, F.A. de Sa, J.L. Santos, E. Joanni. "Optical fober interferometer for measuring the $d_{33}$ coefficient of piezoelectric thin films with compensation of substrate bending". Rev. Sci. Instrum. 73, 2073 (2002).

[12] A. Yariv, P. Yeh, "Optical waves in crystals" (Wiley, New York) (1984).

[13] J.F. Nye. "Physical properties of crystals" (Oxford university Press) (1957).

[14] D.S. Smith, H.D. Riccius, R.P. Edwin. "Refractive indices of lithium niobate". Opt. Commun. 17, 332 (1976).

[15] W.I. Bond. "Measurement of the refractive index of several crystals". J. Appl. Phys. 36, 1674 (1965).

[16] A.W. Warner, M.Onoe, G.A. Coquin. "Determination of elastic and piezoelectric constants for crystals in class (3m)". J. Acoust. Soc. Am. 42, 1223 (1967).

[17] K.F. Hulme, P.H. Davies, V.M. Cound. "The signs of the electro-optic coefficients for lithium niobate". J. Phys. C 2, 855 (1969).

[18] T. Suzuki, O. Eknoyan, H.F. Taylor. "Electrooptic coefficient measurements in $\mathrm{LiTaO}_{3}$ and $\mathrm{LiNbO}_{3}$ waveguides". J. Ligthwave Technol. 11, 285 (1993). 


\begin{tabular}{|l|c|c|}
\hline & $\mathrm{LiNbO}_{3}$ & $\mathrm{LiTaO}_{3}$ \\
\hline Thickness L $(\mathrm{mm})$ & 20 & 12 \\
\hline Inter-electrode distance d $(\mathrm{mm})$ & 5 & 5 \\
\hline $\mathrm{n}_{\mathrm{o}}$ & $2.286[14]$ & $2.177[15]$ \\
\hline $\mathrm{n}_{\mathrm{e}}$ & $2.203[14]$ & $2.181[15]$ \\
\hline $\mathrm{d}_{31}\left(\mathrm{pm} . \mathrm{V}^{-1}\right)$ & $-1[16]$ & $-2[16]$ \\
\hline $\mathrm{r}_{13}^{\mathrm{T}}\left(\mathrm{pm} . \mathrm{V}^{-1}\right)$ & $9.6[17]$ & $8.9[18]$ \\
\hline $\mathrm{r}_{33}^{\mathrm{T}}\left(\mathrm{pm} . \mathrm{V}^{-1}\right)$ & $30.8[17]$ & $32.1[18]$ \\
\hline$\Delta \phi_{\mathrm{o}} / \mathrm{V}\left(\mathrm{mrad} \cdot \mathrm{V}^{-1}\right)$ & 2.22 & 1.04 \\
\hline$\Delta \phi_{\mathrm{e}} / \mathrm{V}\left(\mathrm{mrad} . \mathrm{V}^{-1}\right)$ & 6.49 & 3.91 \\
\hline
\end{tabular}

Table 1 : Parameters of the crystals used in the study 


\begin{tabular}{|l|c|c|c|}
\hline & LTO1 & LTO2 & LTO3 \\
\hline $\begin{array}{l}\text { Sensitivity }\left(\mathrm{mrad} . \mathrm{V}^{-1}\right), \\
\text { ordinary polarisation at } \\
183 \mathrm{~Hz}\end{array}$ & $1.0095 \pm 0.0015$ & $0.9362 \pm 0.0016$ & $1.0486 \pm 0.0014$ \\
\hline $\begin{array}{l}\text { Sensitivity (mrad. } \mathrm{V}^{-1} \text { ), } \\
\text { ordinary polarisation at } \\
25.7 \mathrm{kHz}\end{array}$ & $1.03814 \pm 0.00027$ & $0.99336 \pm 0.00021$ & $0.85363 \pm 0.00022$ \\
\hline $\begin{array}{l}\text { Sensitivity (mrad. } \mathrm{V}^{-1} \text { ), } \\
\text { extraordinary polarisation } \\
\text { at } 25.7 \mathrm{kHz}\end{array}$ & $3.7958 \pm 0.0011$ & $3.5999 \pm 0.0009$ & $3.17205 \pm 0.0010$ \\
\hline Ratio of sensitivities & & 3.624 & \\
\hline
\end{tabular}

Table 2 : Measurement sensitivity of $\mathrm{LiTaO}_{3}$ crystals 


\section{Figure captions}

Figure 1 : Experimental set-up for phase shift measurement. Pol : Polarizer, $\lambda / 2$ : half wave plate, PM : Piezo-mirror, Det : Detector, S : sample. The laser beam is expanded and an image of the output face of the sample is made on the detector.

Figure 2: (a) : Temporal evolution of the mean signal $I_{\text {Mean }}$ and of the RMS value $\Delta I_{R M S}$ of the sinusoidal signal. The piezo mirror is sinusoidally excited and the secondary maximum and minimum peaks of $\mathrm{I}_{\text {Mean }}$ (around 10s and 35s) corresponds to the return points of the displacement of the piezomirror. (b) : Parametric plot of the curve $\Delta I_{R M S}=f\left(I_{\text {Mean }}\right)$

Figure 3 : Evolution of the square of the RMS value of the sinusoidal signal as a function of the mean signal. The line in black is the fit with a parabola (the data are the ones of Figure 2).

Figure 4: Experimental evolution of the phase shift as a function of the applied voltage, with its linear fit, for ordinary polarization (square and dotted line) and extraordinary polarization (circles and full line). In the insert, the data for ordinary polarization are shown in log-log scale. The frequency of the sinusoidal applied voltage is $12.67 \mathrm{kHz}$.

Figure 5: Evolution of the sensitivity of the Pockels $\mathrm{LiNbO}_{3}$ crystal as a function of the frequency of the applied voltage. 


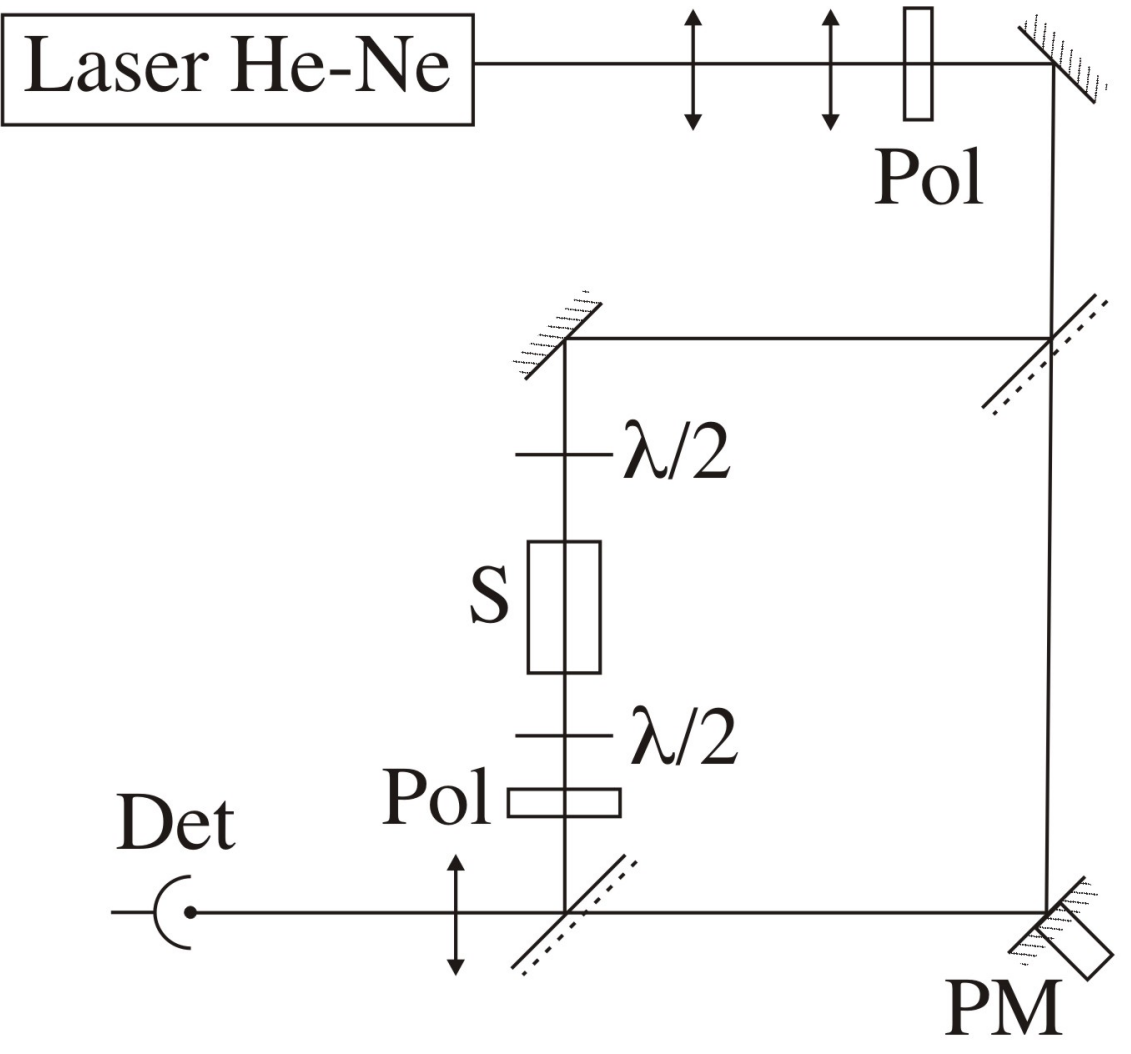

Figure 1 : Delaye et al. 


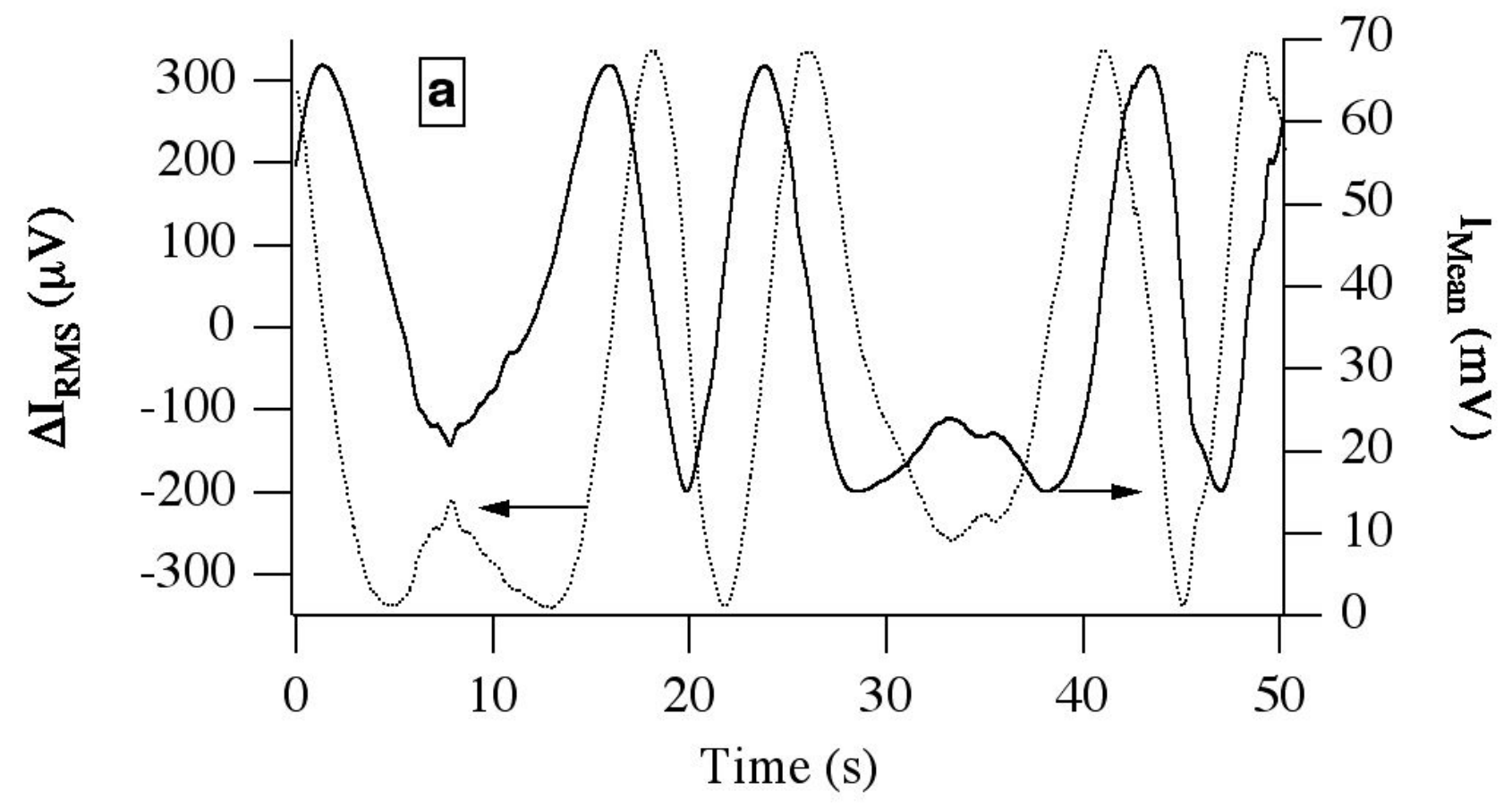

Figure 2a : Delaye et al. 


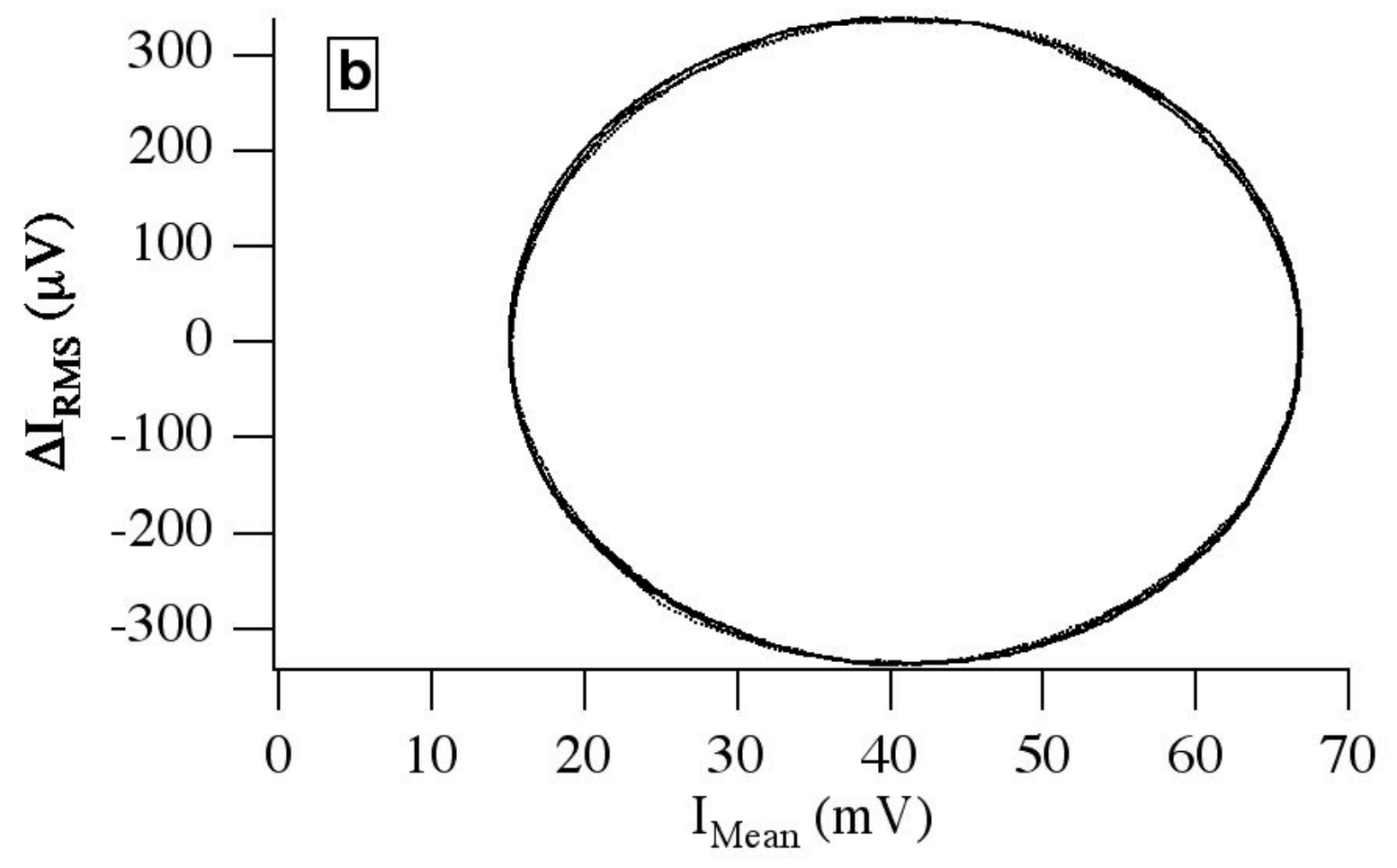

Figure 2b : Delaye et al. 


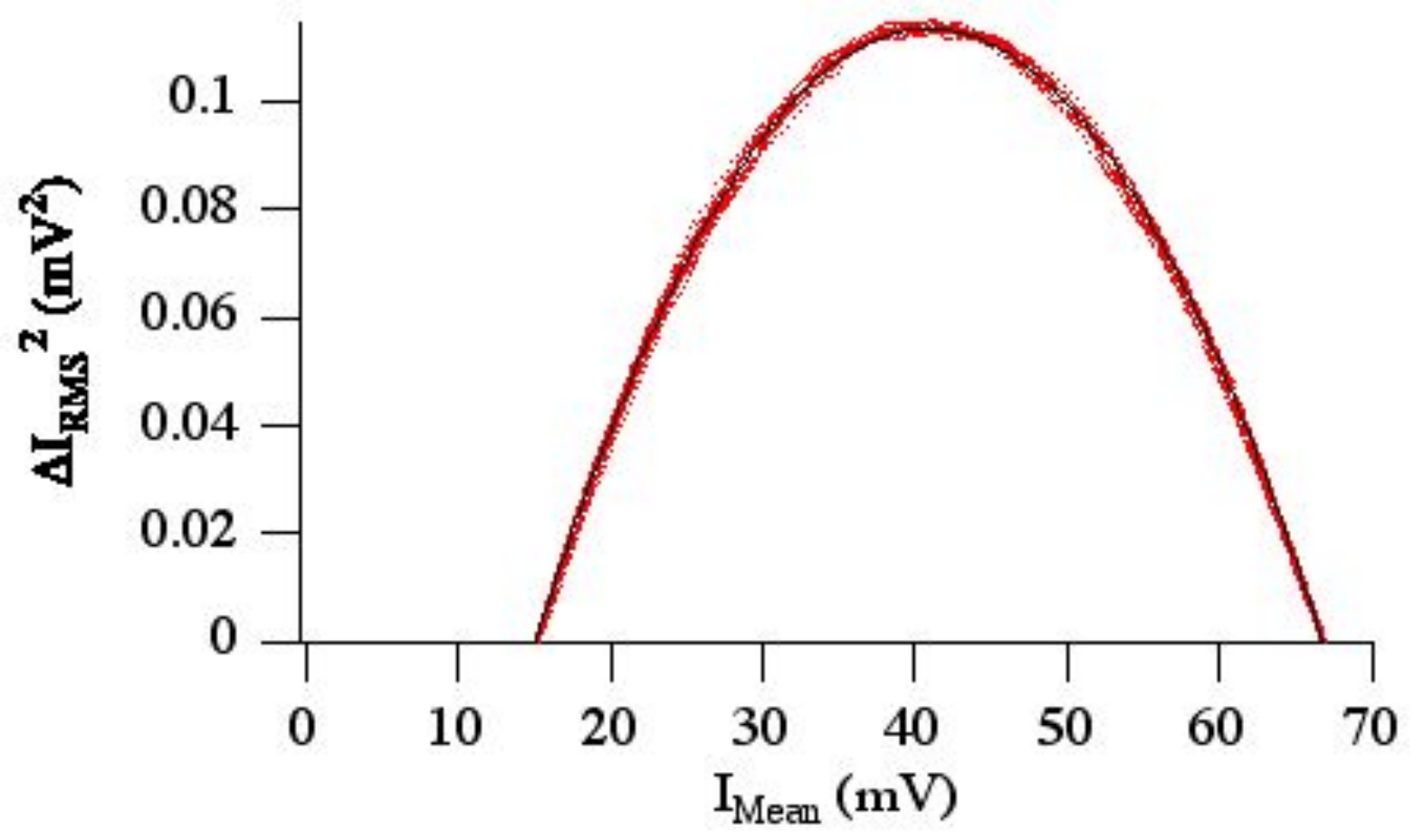

Figure 3 : Delaye et al. 


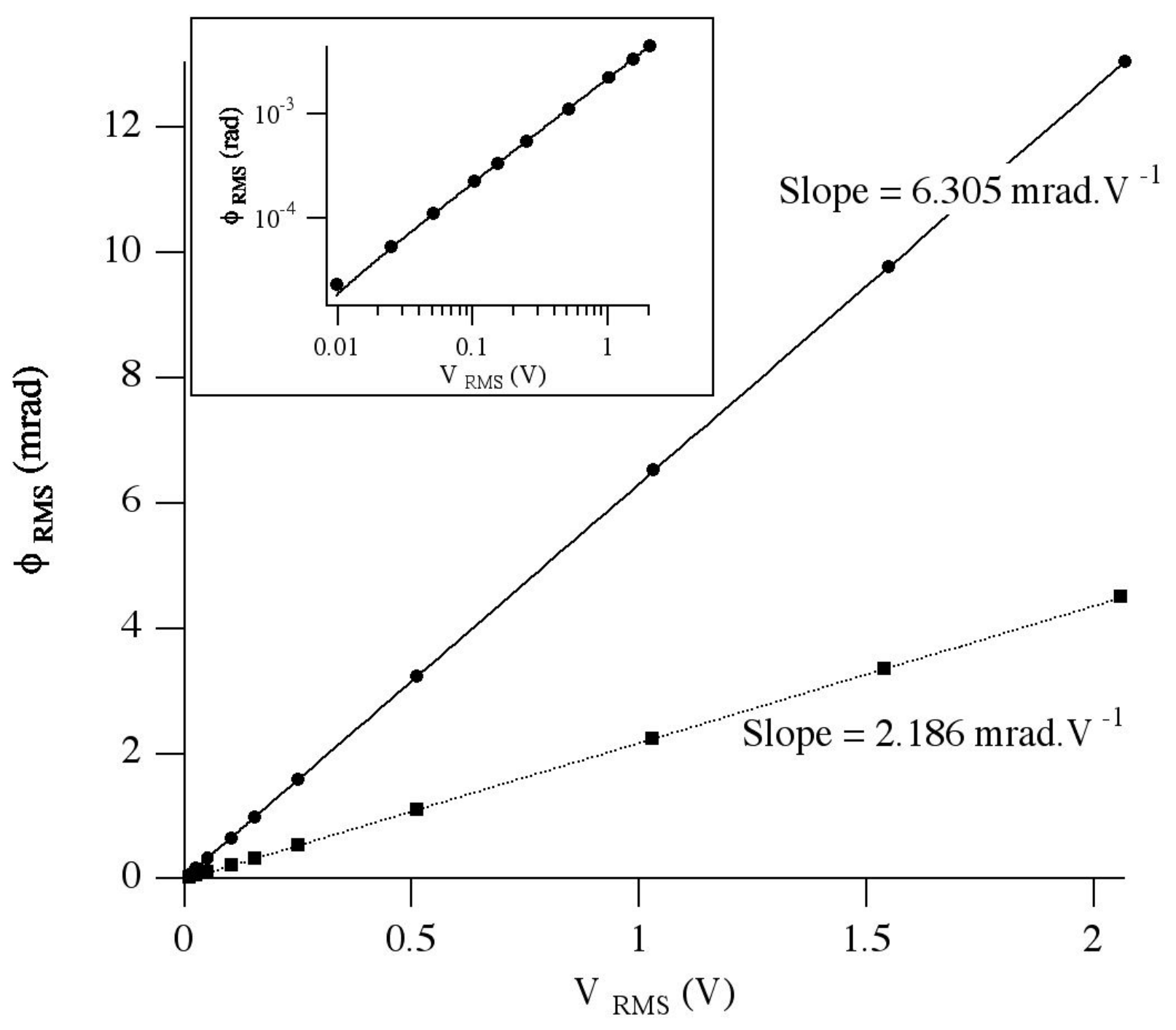

Figure 4 : Delaye et al. 


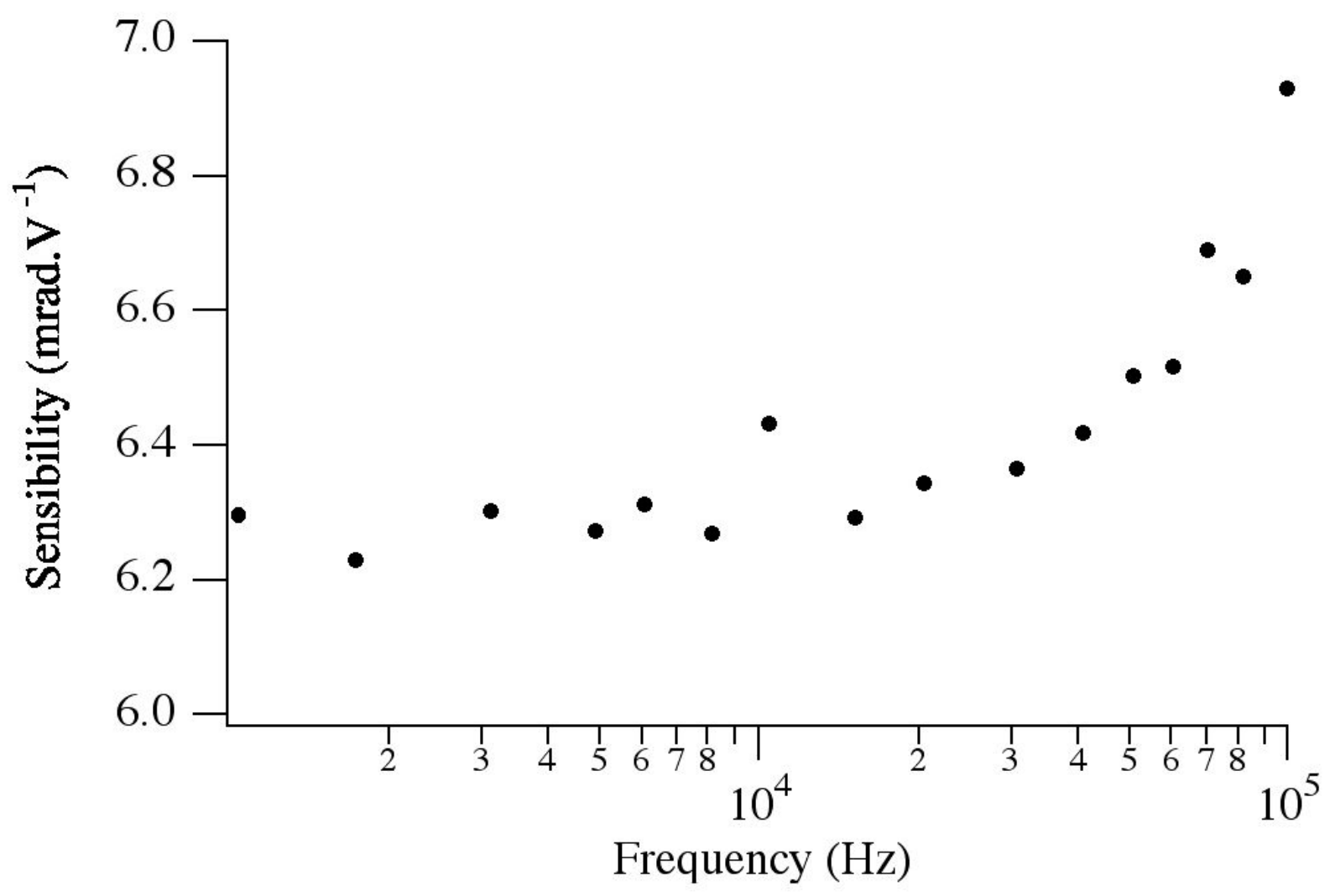

Figure 5 : Delaye et al. 